\title{
Profile of Obesity and Factors Associated Among Adults Cameroonian Living in Urban Area: A Cross Sectional Study
}

\author{
Christine Fernande Nyangono Biyegue ${ }^{1,5, *}$, William Dakam ${ }^{2,5}$, Francoise Raissa Ntentie ${ }^{3,5}$, \\ Nathalie Missia ${ }^{1}$, Jasmine Nika ${ }^{1}$, Marie Noelle Mbemene ${ }^{1}$, Judith Laure Ngondi ${ }^{4,5}$, \\ Julius Enyong Oben ${ }^{4,5}$ \\ ${ }^{1}$ Advanced Teacher Training College for Technical Education, University of Douala, Douala, Cameroon \\ ${ }^{2}$ Faculty of Medicine and Pharmaceuticals Sciences, University of Douala, Douala, Cameroon \\ ${ }^{3}$ Higher Teacher's Training College, University of Maroua, Maroua, Cameroon \\ ${ }^{4}$ Faculty of Sciences, University of Yaounde, Yaounde, Cameroon \\ ${ }^{5}$ Laboratory of Nutrition and Nutritional Biochemistry, University of Yaounde, Yaounde, Cameroon
}

Email address:

spfernande@yahoo.fr (C. F. N. Biyegue)

${ }^{*}$ Corresponding author

\section{To cite this article:}

Christine Fernande Nyangono Biyegue, William Dakam, Francoise Raissa Ntentie, Nathalie Missia, Jasmine Nika, Marie Noelle Mbemene, Judith Laure Ngondi, Julius Enyong Oben. Profile of Obesity and Factors Associated Among Adults Cameroonian Living in Urban Area: A Cross Sectional Study. Central African Journal of Public Health. Vol. 6, No. 5, 2020, pp. 256-267. doi: 10.11648/j.cajph.20200605.14

Received: July 16, 2020; Accepted: August 21, 2020; Published: September 16, 2020

\begin{abstract}
Background and objectives: The emergence of nutritional transition in developing countries leads to increased prevalence of obesity and related adverse health effects. Cameroon which urbanization rate is among the highest in SubSaharan Africa is facing to this situation. This study was aimed at establishing the obesity profile of Cameroonian population living in Douala. Methods: Across-sectional and descriptive survey was performed during July2016 in Douala city. A total of 650 apparently healthy participants aged between 18 years to 60 years were recruited during health campaign organized by the Cameroon Nutritional Science Society on good nutritional practices. The data were collected by using a questionnaire adapted from WHOSTEP wise approach for chronic disease risk factor surveillance. WHO guidelines and NCEP-ATPIII definition were used to define overweight (BMI: $25.0-29.9 \mathrm{~kg} / \mathrm{m}^{2}$ ), Overweight/Obesity (BMI $\geq 25.0 \mathrm{~kg} / \mathrm{m} 2$ ), generalized obesity (GO, BMI $\geq 30.0 \mathrm{~kg} / \mathrm{m} 2$ ), abdominal obesity ( $\mathrm{AO}$, waist circumference $\geq 102 \mathrm{~cm}$ for men and $\geq 88 \mathrm{~cm}$ for women) and combined obesity (CO, GO plus AO). Results: The prevalence of overweight/obesity, GO, AO and CO was 54.2\%, 25.2\%, 40.2\% and $16.2 \%$ respectively. Concerning risk factors: age (38-47 years, female gender, marriage (or in couple) status, secondary level and trade activity were associated to overweight / obesity. Age (28-37 years; 38-47 years and 58 60 years), female gender, marriage (or in couple), primary level, secondary level, and away from home foods consumption, were associated to GO. Age (38-47 years, 48-57 years and 58-60 years), female gender, marriage (or in couple) status, primary level, secondary level, trade activity and cigarette smoking were associated to CO. Risk factors associated to AO were: Age (38-47years), female gender, marriage (or in couple) status, polygamous marriage, secondary level, trade activity two meals/ day consumption, morning and evening meals timing consumption, restaurant and fast-food place of purchase and consumption, daily eating offriedfoods, chocolate consumption, imported distilled beverages consumption and cigarette smoking. Conclusion: All obesity profiles were found among Douala dwellers and AO which is among all an important cardiovascular risk factor was the most frequent. Nutritional education should be emphasized to reduce and prevent complications.
\end{abstract}

Keywords: Abdominal Obesity, Combined Obesity, Generalized Obesity, Douala 


\section{Introduction}

Obesity is defined as an abnormal growth of adipose tissue because of an enlargement of adipose cell (hypertrophic obesity) or an increase in number of adipose cells (hyperplasic obesity) or a combination of both [1]. Regardless of fat accumulation, they are android or abdominal obesity with abdominal fat distribution, and gynoid obesity with peripheral fat accumulation. The most harmful remains the abdominal one [1].

According to WHO, more than 1.9 billion adults aged of 18 years and above were overweight and more than 650 million were obese. Obesity represents a rapidly growing threat to the health of populations of many countries. Indeed, they are now so common that they are replacing more traditional problems such as under nutrition and infectious diseases as the most significant causes of morbidity [2]. Obesity is associated with the incidence of multiple comorbidities including type II diabetes, cancer and cardiovascular diseases [3]. Individuals with obesity have higher rates of mortality and morbidity compared to non obese individuals [4].

Obesity is generally classified as generalized obesity (GO) and abdominal obesity (AO) [5]. Abdominal obesity defined by WC or WHtR more effectively reflects the visceral fat and has been shown to be more strongly associated than BMI with CVDs and overall mortality [6-8].

Fat accumulation is associated with numerous comorbidities such as cardiovascular diseases (CVD), sleep disorders and gall bladder diseases [9]. For CVD, fat accumulation affects the risk of major outcomes, both directly and indirectly through its links with risk factors such as hypertension, type 2 diabetes, insulin resistance, lipid disorders and other metabolic and hematological abnormalities [10].

The growing worldwide problem of overweight, obesity, and diet-related chronic diseases in the face of persistent poor- quality diets is thought to be driven by urbanization and globalization [11]. Obesity is more common in Africa and is related to rapid urbanization and socio-economic development which lead to a westernization of lifestyle through the adoption of higher energy density diet and sedentarity [12]. These urban components make the modern city obesogenic [13], and therefore associated with increasing rates of non-communicable diseases (NCDs), such as hypertension and type 2 diabetes [14].

Cameroon, like other LMICs, is currently undergoing a socio-economic transition characterised by improving standards of living, rapid (mostly unplanned) urbanisation and westernisation of lifestyles, including increased tobacco use, unhealthy diet, insufficient physical activity and the harmful use of alcohol [15]. These changes are accompanied by changes in the distribution and patterns of disease; with NCDs increasingly contributing a significant share of the public health burden of disease, alongside an unfinished agenda of infections. The urbanisation and social mobility that accompanied economic development led to the increase in obesity, diabetes and hypertension with a higher prevalence in urban areas than in rural areas [15].

Adequate management of obesity as a chronic condition for those who are already obese is important and requires the principles of integrated care for disease management. The upstream causal factors need first to be identified [16]. Factors in the global food system combined with local environmental factors result in large differences in obesity prevalence between populations [17].

The aim of this study was to assess the profile of obesity encountered among adults in urban area and establish the association with sociodemographic and lifestyle factors.

\section{Methodology}

A cross-sectional and descriptive survey was performed in Douala city. A total of 650 apparently healthy participants aged between 18 to 60 years were recruited during health campaign organized by the Cameroon Nutritional Science Society on good nutritional practices. Cameroonian who live in Douala since at least 6 months were included in the sample study. Pregnant or lactating women, people with physical and mental disabilities as well as non-consenting participants, were excluded.

\subsection{Data Collection}

Data were collected using questionnaire adapted from WHO STEPwise approach for chronic disease risk factor surveillance. Informations on age, gender, education level, marital status, regime of marriage, source of incomes, and dietary behaviour factors were collected.

\subsection{Anthropometric Measurements}

Body weight was measured to the nearest $100 \mathrm{~g}$, with participants in light clothing and without shoes, using a portable electronic scale of $150 \mathrm{~kg}$ capacity $\left(\mathrm{TANITA}^{\mathrm{TM}}\right.$ ). Height was measured to the nearest $0.5 \mathrm{~cm}$ using a portable locally built stadiometer, with the participants standing upright on a flat surface without shoes and with the back of their heels and the occiput against the stadiometer. Waist circumference which (WC) is strongly correlated to visceral adipose tissue and associated to several cardiometabolic risk factors was measured [19]. It was measured to the nearest $0.1 \mathrm{~cm}$ with a flexible, non-stretchable and tension-regulated steel tape, at the midpoint between the lowest rib and the iliac crest, while participants were standing and breathing normally.

BMI was calculated as weight (in kilograms) divided by the square of height (in metres). BMI was categorised as follows [20]: Normal weight, $B M I<24.9 \mathrm{~kg} / \mathrm{m}^{2}$; overweight, BMI: $25.0-29.9 \mathrm{~kg} / \mathrm{m}^{2}$; obesity, BMI $\geq 30.0 \mathrm{~kg} / \mathrm{m}^{2}$. Abdominal obesity was defined as $\mathrm{WC} \geq 102 \mathrm{~cm}$ for men and $\mathrm{WC} \geq 88 \mathrm{~cm}$ for women [21].

\subsection{Definitions of Obesity Profile}

The modified definition of [21] has been used to define several types of obesity. The initial definition is based on the 
World Health Organization Asia Pacific Guidelines. In our study this definition has been modified by using the world WHO guidelines to define overweight and obesity. Thus:

Overweight was defined as $\mathrm{BMI} \geq 25.0 \mathrm{~kg} / \mathrm{m}^{2}$ but $<30.0 \mathrm{~kg} / \mathrm{m}^{2}$ for both genders with or without abdominal obesity;

Generalized obesity (GO) was defined as $\mathrm{BMI} \geq 30.0 \mathrm{~kg} / \mathrm{m}^{2}$ for both genders with or without abdominal obesity;

Abdominal obesity (AO) was defined as $\mathrm{WC} \geq 102 \mathrm{~cm}$ for men and $\mathrm{WC} \geq 88 \mathrm{~cm}$ for women with or without generalized obesity;

Combined obesity $(\mathrm{CO})$ : individuals with both $\mathrm{GO}$ and $\mathrm{AO}$

Overweight/obesity was defined as $\mathrm{BMI} \geq 25.0 \mathrm{~kg} / \mathrm{m}^{2}$ [19].

\subsection{Variables}

In this study, the outcome variables were overweight/obesity, GO, AO, and CO.

Independent variables were age, gender, socioeconomic conditions (education, marital status, type of marriage and sources of income) and lifestyle (smoking, alcohol, lifestyle behaviour factors and dietary habits). The hypotheses were that the above variables are associated with overweight/obesity, GO, AO or CO.

Age (Years) as a continuous variable, we classified into 5 groups: $18-27,28-37,38-47,48-57$ and 58-60.

Whilst the highest completed level of education was obtained as none education, primary level, secondary level and university level.

Participants' eating habits were explored through interview. For each participant, we documented over a period of past seven days informations about frequency of "traditional" foods consumption, foods specific to the "urban" environment and "western" foods. We also collected data on the frequency of away from home foods consumption or alcohol, Consumption habits; type of drink (wine, beer, liqueurs) and their origin (local or imported) have been documented. Participants were ranked according to whether they consumed alcohol or not.

\subsection{Ethical Considerations}

This study is a part of project intitled "Nutrition and
Health study" approved the National Ethics Committee $\mathrm{N}^{\circ} 2014 / 08 / 488 / \mathrm{EC} / \mathrm{CNERSH}$. It was conducted in strict compliance with the physical, moral and psychological integrity of all participants; following the principles outlined in the Helsinki Declaration. Prior to participant enrolment, they were informed about the purpose of the study and data collection procedures and all volunteer participants signed an informed consent form.

\subsection{Datas Analysis}

Data were analyzed using IBM SPSS version 22.0. Data were described using frequency distributions, measures of central tendency and dispersion. Factors association with overweight/obesity and other forms of obesity were identified using binary logistic regression analyses. Unadjusted odds ratio (OR) and their 95\% confidence intervals (CI) were reported. Proportions were estimated using the Yates corrected Chi-square test and $\mathrm{p}$ value of less than $5 \%$ indicated a significant difference between two proportions.

\section{Results}

The sample included 650 adults aged $18-60$ years $(35.2 \%$ men versus $64.8 \%$ women) and about half of the participants were within age ranged $25-34$ years $(45.6 \%)$.

Table1 recording anthropometric characteristic and the prevalence of overweight and obesity in study population. The results of anthropometric characteristic show that women had lower mean age and height than men $(p<0.01)$ but higher mean BMI values than men $(\mathrm{p}<0.01)$.

Concerning the overall prevalence of overweight and obesity in general population. The prevalence of overweight, $\mathrm{GO}, \mathrm{AO}$ and $\mathrm{CO}$ was $29.2 \%$, (38.4\% in men versus $61.6 \%$ in women) $25.2 \%$ (21.3\% in men versus $78.7 \%$ in women), $40.2 \%(8.4 \%$ in men versus $91.6 \%$ in women) and $16.2 \%$ (9.5\% in men versus $90.5 \%$ in women) respectively. The cumulated prevalence of overweight and obesity was $54.5 \%$ (30.5\% in men versus $69.5 \%$ in women). In general this prevalence is higher in women than men.

Table 1. Anthropometric characteristics and prevalence of overweight and obesity of the study population.

\begin{tabular}{llll}
\hline Variables & Total population $(\mathbf{n = 6 5 0})$ & Men $(\mathbf{n = 2 2 9})$ & Women $(\mathbf{n = 4 2 1})$ \\
\hline Age $($ years $)$ & $34.72 \pm 0.41$ & $36.54 \pm 0.72$ & $33.72 \pm 0.50^{* *}$ \\
Height $(\mathrm{m})$ & $1.65 \pm 0.004$ & $1.69 \pm 0.005$ & $1.62 \pm 0.005^{* *}$ \\
Weight $(\mathrm{Kg})$ & $72.93 \pm 0.59$ & $73.03 \pm 0.87$ & $72.88 \pm 0.78$ \\
BMI $\left(\mathrm{kg} / \mathrm{m}^{2}\right)$ & $26.89 \pm 0.24$ & $25.49 \pm 0.27$ & $27.66 \pm 0.33^{* *}$ \\
Waist circumference $(\mathrm{cm})$ & $84.85 \pm 0.80$ & $85.21 \pm 1.21$ & $84.66 \pm 1.06$ \\
Overweight (n (\%)) & $190(29.2)$ & $73(38.4)$ & $117(61.6)$ \\
Generalized obesity (GO) (n (\%)) & $164(25.2)$ & $35(21.3)$ & $129(78.7)$ \\
Overweight/obesity (O/O) (n (\%)) & $354(54.5)$ & $108(30.5)$ & $246(69.5)$ \\
Abdominal obesity (AO) (n (\%)) & $261(40.2)$ & $22(8.4)$ & $239(91.6)$ \\
Combined obesity (CO) (n (\%)) & $105(16.2)$ & $10(9.5)$ & $95(90.5)$ \\
\hline
\end{tabular}

** means significantly different between men and women at $\mathrm{p}<0.01$.

Table 2 represents prevalence of obesity according to age among gender (women and men).
The specific results of the prevalence of obesity among women and men revealed a high prevalence with advanced in 
age (table 2), lower prevalence among 18-27years and higher prevalence among 38-47 years in all type of obesity. In

general, the prevalence of $\mathrm{O} / \mathrm{O}$ is high in all age categories.

Table 2. Prevalence of types of obesity according to age among women and men.

\begin{tabular}{llllll}
\hline $\begin{array}{l}\text { Age categories } \\
\text { (years) }\end{array}$ & Women & TO $(\mathbf{n}=\mathbf{1 2 9}) \mathbf{n}(\mathbf{\%})$ & $\mathbf{O} / \mathbf{O}(\mathbf{n}=\mathbf{2 4 6}) \mathbf{n}(\%)$ & $\mathbf{A O}(\mathbf{n}=\mathbf{2 3 9}) \mathbf{n}(\%)$ & $\mathbf{C O}(\mathbf{n}=\mathbf{9 5}) \mathbf{n}(\mathbf{( \% )}$ \\
\cline { 2 - 6 } & Total population $(\mathbf{n}=\mathbf{4 2 1}) \mathbf{n}(\mathbf{\%})$ & $17(13.3)$ & $58(45.3)$ & $61(47.7)$ & $8(6.3)$ \\
$18-27$ & $128(30.4)$ & $48(28.2)$ & $94(55.3)$ & $91(53.5)$ & $31(18.2)$ \\
$28-37$ & $170(40.3)$ & $35(49.3)$ & $54(76.1)$ & $51(71.8)$ & $31(43.7)$ \\
$38-47$ & $71(16.9)$ & $19(54.3)$ & $26(74.3)$ & $23(65.7)$ & $17(48.6)$ \\
$48-57$ & $35(8.3)$ & $10(58.8)$ & $14(82.4)$ & $13(76.5)$ & $8(47.1)$ \\
$58-60$ & $17(4.0)$ &
\end{tabular}

Table 2. Continued.

\begin{tabular}{|c|c|c|c|c|c|}
\hline \multirow{2}{*}{$\begin{array}{l}\text { Age categories } \\
\text { (years) }\end{array}$} & \multicolumn{5}{|l|}{ Men } \\
\hline & Total population $(n=229) n(\%)$ & GO $(n=35) n(\%)$ & $\mathrm{O} / \mathrm{O}(\mathrm{n}=108) \mathrm{n}(\%)$ & AO $(n=22) n(\%)$ & $\operatorname{CO}(n=10) n(\%)$ \\
\hline $18-27$ & $61(26.6)$ & $4(6.6)$ & $20(32.8)$ & $1(1.6)$ & $1(1.6)$ \\
\hline $28-37$ & 73 (31.9) & $14(19.2)$ & 34 (46.6) & $4(5.5)$ & $2(2.7)$ \\
\hline $38-47$ & $50(21.8)$ & $10(20.0)$ & $32(64.0)$ & $12(24.0)$ & $5(10.0)$ \\
\hline $48-57$ & $35(15.3)$ & $5(14.3)$ & $17(48.6)$ & $4(11.4)$ & $1(2.9)$ \\
\hline $58-60$ & $10(4.4)$ & $2(20.0)$ & $5(50.0)$ & $1(10.0)$ & $1(10.0)$ \\
\hline
\end{tabular}

Sociodemographic factors in relation with obesity

Table 3 presents association between obesity profile and various sociodemographic factors. According to age, we observed that the older the age, the higher the prevalence of obesity. Regardless of the types of obesity, the prevalence was significantly low $(\mathrm{p}<0.01)$ among $18-27$ years but high among 38-47years and 58-60years.

According to gender, the prevalence of overweight was higher among men $(31.9 \%)$ than women $(27.8 \%)(p<0.01)$. However, the cumulative prevalence of overweight /obesity, $\mathrm{GO}, \mathrm{AO}$ and $\mathrm{CO}$ was higher in women than in men $(\mathrm{p}<0.01)$ (table 3).

Concerning the other sociodemographic factors, the results revealed that the prevalence of abdominal obesity was significantly $(\mathrm{p}<0.01)$ high among individuals married to polygamous regime $(53.1 \%)$. In the same way, regardless the types of obesity, the prevalence was low $(p<0.01)$ among single individuals. The prevalence of obesity was high among married individuals (or in couple) and widowed, divorced or separated.

Illiterates individuals were the less vulnerable to all types of obesity $(p<0.01)$. Meanwhile those who attended secondary level of education were more affected by overweight/obesity. In contrary, those who attended primary school were the most affected by all type of obesity. Concerning source of incomes, $\mathrm{AO}$ was significantly higher among $(\mathrm{p}<0.05)$ traders $(50 \%)$.

Table 3. Prevalence of obesity and subtypes according to some sociodemographic factors.

\begin{tabular}{|c|c|c|c|c|c|c|}
\hline $\begin{array}{l}\text { Sociodemographic } \\
\text { characteristics }\end{array}$ & Classification & $\begin{array}{l}\text { Total population } \\
(n=650) \text { n }(\%)\end{array}$ & $\begin{array}{l}\text { Overweight/obesity } \\
(n=354) n(\%)\end{array}$ & $\begin{array}{l}\text { GO }(n=164) \\
n(\%)\end{array}$ & $\begin{array}{l}\text { AO }(n=261) \\
n(\%)\end{array}$ & $\begin{array}{l}\text { CO }(n=105) \\
\text { n }(\%)\end{array}$ \\
\hline \multirow{5}{*}{$\begin{array}{l}\text { Age categories } \\
\text { (years) }\end{array}$} & $18-27$ & $189(29.1)$ & $21(11.1)^{* *}$ & $78(41.3)^{* *}$ & $62(32.8)^{* *}$ & $9(4.8)^{* *}$ \\
\hline & $28-37$ & $243(37.4)$ & $62(25.5)$ & $128(52.7)$ & $95(39.1)$ & $33(13.6)$ \\
\hline & $38-47$ & $121(18.6)$ & $45(37.2)$ & $86(71.1)$ & $63(52.1)$ & $36(29.8)$ \\
\hline & $48-57$ & $70(10.8)$ & $24(34.3)$ & $43(61.4)$ & $27(38.6)$ & $18(25.7)$ \\
\hline & $58-60$ & $27(4.2)$ & $12(44.4)$ & $19(70.4)$ & $14(51.9)$ & $9(33.3)$ \\
\hline \multirow{2}{*}{ Gender } & Men & $229(35.2)$ & $35(15.3)$ & $108(47.2)$ & $22(9.6)$ & $10(4.4)$ \\
\hline & Women & $421(64.8)$ & $129(30.6)^{* *}$ & $246(58.4)^{* *}$ & $239(56.8)^{* *}$ & $95(22.6)^{* *}$ \\
\hline \multirow{2}{*}{ regime of marriage } & Monogamous & $520(80.0)$ & $279(53.7)$ & $132(25.4)$ & $192(36.9)$ & $78(15.0)$ \\
\hline & Polygamous & $130(20.0)$ & $75(57.7)$ & $32(24.6)$ & $69(53.1)^{* *}$ & $27(20.8)$ \\
\hline \multirow{3}{*}{ Marital status } & Marriage or concubinage & $379(58.3)$ & $205(54.1)$ & $108(28.5)$ & $190(50.1)$ & $79(20.8)$ \\
\hline & Widowed, divorced or separated & $39(6.0)$ & $27(69.2)$ & $12(30.8)$ & $20(51.3)$ & $6(15.4)$ \\
\hline & Single & $232(35.7)$ & $122(52.6)^{* *}$ & $44(19.0)^{*}$ & $51(22.0)^{* *}$ & $20(8.6)^{* *}$ \\
\hline \multirow{3}{*}{ Education level } & Illeteracy & $56(8.6)$ & $24(42.9)^{* *}$ & $5(8.9)^{* *}$ & $14(25.0)^{* *}$ & $4(7.1)^{* *}$ \\
\hline & Secondary & $317(48.8)$ & $194(61.2)$ & $96(30.3)$ & $140(44.2)$ & $61(19.2)$ \\
\hline & University & $202(31.1)$ & $94(46.5)$ & $32(15.8)$ & $72(35.6)$ & $19(9.4)$ \\
\hline \multirow{5}{*}{ Source of incomes } & Permanent salary & $192(29.5)$ & $98(51.0)$ & $43(22.4)$ & $77(40.1)$ & $27(14.1)$ \\
\hline & Temporarysalary & $60(9.2)$ & $33(55.0)$ & $10(16.7)$ & $16(26.7)$ & $4(6.7)$ \\
\hline & Trade & $176(27.1)$ & $107(60.8)$ & $49(27.8)$ & $88(50.0)^{*}$ & $38(21.6)$ \\
\hline & Help/assistance & $53(8.2)$ & $24(45.3)$ & $11(20.8)$ & $18(34.0)$ & $8(15.1)$ \\
\hline & Spouse & $169(26.0)$ & $92(54.4)$ & $51(30.2)$ & $62(36.7)$ & $28(16.6)$ \\
\hline
\end{tabular}

** means $\mathrm{p}<0.01$; * means $\mathrm{p}<0.05$ between different groups of each variable. 
Risk factors associated with obesity

Sociodemographic factors associated to obesity

Table 4 shows simple logistic regression analysis using overweight/obesity, GO, $\mathrm{AO}$ and $\mathrm{CO}$ as the dependent variables and socio-demographic factors as independent variables. Based on odds ratio, it appears that female gender versus male, had 1.45 (95\% CI: 1.03-2.03) times increased odds of overweight/obesity, 2.41 (95\% CI: 1.59-3.66) times increased odds of GO, 14.48 (95\% CI: 8.74-23.99) times increased odds of AO and 6.06 (95\% CI: 3.08-11.91) times increased odds of CO. Marriage (or in couple) status were also significantly associated with overweight/obesity [1.38 (95\% CI: 1.01-1.89)], GO [1.53 (95\% CI: 1.06-2.21)], AO [2.83 (95\% CI: 2.02-3.97)] and CO [2.48 (95\% CI: 1.54 3.99)].

With 18-27 years old as reference group, age 38-47 was significantly associated to overweight/obesity $[2.40$ (95\% CI:
1.56-3.67)], GO [2.04 (95\% CI: 1.34-3.11)], AO [1.82 (95\% CI: $1.22-2.70)]$ and CO [2.82 (95\% CI: 1.77-4.49)]. Also, age 28-37 [1.02 (95\% CI: 1.56-3.67)] and 58-60 [2.48 (95\% CI: 1.14-5.41)] were significantly associated with GO while 48-57 [1.96 (95\% CI: 1.10-3.51)] and 58-60 [2.75 (95\% CI: 1.20-6.29)] were associated with CO. Polygamous marriage increased 1.82 (95\% CI: 1.14-2.90) times AO.

Concerning level of education, secondary $[1.71$ (95\% CI: 1.25-2.33)] level was associated with overweight/obesity. On the other hand, primary and secondary levels were significantly associated with GO and $\mathrm{CO}$ while secondary level [1.39 (95\% CI: 1.01-1.90)] is only associated with AO compared to other groups. Trade activities were significantly associated to overweight/obesity [1.43 (95\% CI: 1.00-2.03)], AO [1.74 (95\% CI: 1.23-2.47)] and CO [1.73 (95\% CI: $1.08-2.60)]$.

Table 4. Simple logistic regression of obesity with socio-demographic factors.

\begin{tabular}{|c|c|c|c|c|c|}
\hline \multirow{3}{*}{$\begin{array}{l}\text { Sociodemographic } \\
\text { characteristics } \\
\text { (reference) }\end{array}$} & \multirow{3}{*}{ Classification } & Overweight/obesity & GO & AO & $\mathrm{CO}$ \\
\hline & & Odds ratio $(95 \% \mathrm{CI})$ & Odds ratio $(95 \% \mathrm{CI})$ & Odds ratio $(95 \% \mathrm{CI})$ & Odds ratio $(95 \% \mathrm{CI})$ \\
\hline & & P value & P value & P value & P value \\
\hline \multirow{7}{*}{$\begin{array}{l}\text { Age category (years) } \\
\text { (18-27 years) }\end{array}$} & \multirow{2}{*}{ 28-37 years } & $0.89(0.65-1.23)$ & $1.02(0.71-1.48)$ & $0.93(0.67-1.29)$ & $0.73(0.47-1.14)$ \\
\hline & & $>0.05$ & $<0.01$ & $>0.05$ & $>0.05$ \\
\hline & \multirow{2}{*}{$38-47$ years } & $2.40(1.56-3.67)$ & $2.04(1.34-3.11)$ & $1.82(1.22-2.70)$ & $2.82(1.77-4.49)$ \\
\hline & & $<0.01$ & $<0.01$ & $<0.01$ & $<0.01$ \\
\hline & $48-57$ years & $1.38(0.83-2.29)$ & $1.64(0.97-2.78)$ & $0.93(0.56-1.55)$ & $1.96(1.10-3.51)$ \\
\hline & \multirow{2}{*}{$58-60$ years } & $2.04(0.88-4.73)$ & $2.48(1.14-5.41)$ & $1.64(0.76-3.55)$ & $2.75(1.20-6.29)$ \\
\hline & & $>0.05$ & $<0.05$ & $>0.05$ & $<0.05$ \\
\hline \multirow{2}{*}{ Gender (Men) } & \multirow{2}{*}{ Women } & $1.45(1.03-2.03)$ & $2.41(1.59-3.66)$ & $14.48(8.74-23.99)$ & $6.06(3.08-11.91)$ \\
\hline & & $<0.05$ & $<0.05$ & $<0.05$ & $<0.05$ \\
\hline \multirow{2}{*}{$\begin{array}{l}\text { regime of marriage } \\
\text { (monogamous) }\end{array}$} & \multirow{2}{*}{ Polygamous } & $1.18(0.80-1.74)$ & $0.96(0.62-1.50)$ & $1.82(1.14-2.90)$ & $1.49(0.91-2.42)$ \\
\hline & & $>0.05$ & $>0.05$ & $<0.05$ & $>0.05$ \\
\hline \multirow{2}{*}{ Marital status (Single) } & Marriage or in couple & $\begin{array}{l}1.38(1.01-1.89) \\
<0.05\end{array}$ & $\begin{array}{l}1.53(1.06-2.21) \\
<0.01\end{array}$ & $\begin{array}{l}2.83(2.02-3.97) \\
<0.01\end{array}$ & $\begin{array}{l}2.48(1.54-3.99) \\
<0.01\end{array}$ \\
\hline & $\begin{array}{l}\text { Widowed, divorced or } \\
\text { separated }\end{array}$ & $>0.05$ & $>0.05$ & $>0.05$ & $>0.05$ \\
\hline \multirow{5}{*}{$\begin{array}{l}\text { Level of education } \\
\text { (illeteracy) }\end{array}$} & Primary & $\begin{array}{l}1.07(0.66-1.74) \\
>0.05\end{array}$ & $\begin{array}{l}2.34(1.42-3.86) \\
<0.01\end{array}$ & $\begin{array}{l}1.35(0.83-2.20) \\
>0.05\end{array}$ & $\begin{array}{l}2.27(1.31-3.96) \\
<0.01\end{array}$ \\
\hline & \multirow{2}{*}{ Secondary } & $1.71(1.25-2.33)$ & $1.69(1.18-2.42)$ & $1.39(1.01-1.90)$ & $1.57(1.03-2.39)$ \\
\hline & & $<0.01$ & $<0.01$ & $<0.05$ & $<0.05$ \\
\hline & \multirow{2}{*}{ University } & $0.63(0.45-0.88)$ & $0.45(0.29-0.69)$ & $0.76(0.54-1.07)$ & $0.44(0.26-0.74)$ \\
\hline & & $<0.01$ & $<0.01$ & $>0.05$ & $<0.01$ \\
\hline \multirow{8}{*}{$\begin{array}{l}\text { Source of incomes } \\
\text { (Temporary salary) }\end{array}$} & \multirow{2}{*}{ Permanent salary } & $0.82(0.59-1.15)$ & $0.80(0.54-1.20)$ & $0.99(0.71-1.41)$ & $0.80(0.50-1.28)$ \\
\hline & & $>0.05$ & $>0.05$ & $>0.05$ & $>0.05$ \\
\hline & \multirow{2}{*}{ Trade } & $1.43(1.00-2.03)$ & $1.204(0.82-1.78)$ & $1.74(1.23-2.47)$ & $1.73(1.08-2.60)$ \\
\hline & & $<0.05$ & $>0.05$ & $<0.01$ & $<0.05$ \\
\hline & \multirow{2}{*}{ Help/assistance } & $0.67(0.38-1.18)$ & $0.76(0.38-1.51)$ & $0.75(0.42-1.35)$ & $0.92(0.42-2.01)$ \\
\hline & & $>0.05$ & $>0.05$ & $>0.05$ & $>0.05$ \\
\hline & \multirow{2}{*}{ Spouse } & $1.0(0.70-1.42)$ & $1.41(0.95-2.08)$ & $0.82(0.57-1.18)$ & $1.04(0.65-1.67)$ \\
\hline & & $>0.05$ & $>0.05$ & $>0.05$ & $>0.05$ \\
\hline
\end{tabular}

\section{Practical lifestyle habits associated to obesity}

Table 5 show simple logistic regression using overweight/obesity, GO, AO and $\mathrm{CO}$ as dependent variables and practical lifestyle habits as independent variables.

Consumption of 2 meals per day increased 1.51 (1.06-2.15) times the risk of AO. Concerning meal times per day, it appears that the consumption of meals morning and evening increased 3.12 (95\% CI: 1.99-4.89) times the risk of AO while the consumption of meals morning, noon and evening protected against AO [0.49 (95\% CI: 0.6-0.68)].

Away from home foods consumption increased risk of GO by 1.69 (95\% CI: 1.07-2.68) times. Meanwhile, eating in restaurant [1.67 (95\% CI: 1.21-2.29)], and fast food [2.36 (95\% CI: $1.32-$ 4.24)] were associated with increased risk of AO.

The daily consumption of fried foods increased by 2.74 (95\% CI: 1.76-4.26) times the risk of AO meanwhile about once a week consumption was protective 0.60 (95\% CI: $0.43-0.82)$. 
Table 5. Simple logistic regression of obesity with the meals intake, the consumption of food outside in the home, places of purchase and fried foods consumption.

\begin{tabular}{|c|c|c|c|c|c|}
\hline \multirow[b]{2}{*}{ Variables (reference) } & & Overweight/obesity & GO & AO & $\mathrm{CO}$ \\
\hline & & Odds ratio $(95 \% \mathrm{CI})$ & Odds ratio $(95 \% \mathrm{CI})$ & Odds ratio $(95 \% \mathrm{CI})$ & Odds ratio $(95 \% \mathrm{CI})$ \\
\hline \multirow{5}{*}{$\begin{array}{l}\text { Number of meals per day } \\
(3 \text { meals })\end{array}$} & \multirow{3}{*}{2} & P vilue & Pvalue & Paine & \\
\hline & & $0.61(0.43-0.87)$ & $0.70(0.48-1.02)$ & $1.51(1.06-2.15)$ & $0.98(0.62-1.54)$ \\
\hline & & $<0.01$ & $>0.05$ & $<0.05$ & $>0.05$ \\
\hline & \multirow{2}{*}{4} & $1.27(0.85-1.90)$ & $1.22(0.76-1.97)$ & $0.53(0.35-0.79)$ & $0.92(0.54-1.57)$ \\
\hline & & $>0.05$ & $>0.05$ & $<0.01$ & $>0.05$ \\
\hline \multirow{4}{*}{$\begin{array}{l}\text { Meals times per day } \\
\text { (Noon) }\end{array}$} & \multirow{3}{*}{$\begin{array}{l}\text { Morning + } \\
\text { evening } \\
\text { Morning +noon } \\
+ \text { evening }\end{array}$} & $\begin{array}{l}1.35(0.92-1.98) \\
>0.05\end{array}$ & $\begin{array}{l}0.74(0.49-1.13) \\
>0.05\end{array}$ & $\begin{array}{l}3.12(1.99-4.89) \\
<0.01\end{array}$ & $\begin{array}{l}1.53(0.87-2.72) \\
>0.05\end{array}$ \\
\hline & & $1.18(0.86-1.60)$ & $1.13(0.80-1.61)$ & $0.49(0.36-0.68)$ & $0.70(0.46-1.07)$ \\
\hline & & $>0.05$ & $>0.05$ & $<0.01$ & $>0.05$ \\
\hline & Noon +evening & $\begin{array}{l}1.08(0.66-1.76) \\
>0.05\end{array}$ & $\begin{array}{l}1.10(0.62-1.96) \\
>0.05\end{array}$ & $\begin{array}{l}0.68(0.42-1.11) \\
>0.05\end{array}$ & $\begin{array}{l}1.08(0.55-2.12) \\
>0.05\end{array}$ \\
\hline \multirow{3}{*}{$\begin{array}{l}\text { Away-from-home foods } \\
\text { consumption (No) }\end{array}$} & \multirow{2}{*}{ Yes } & $1.28(0.83-1.98)$ & $1.69(1.07-2.68)$ & $0.75(0.48-1.17)$ & $1.61(0.95-2.73$ \\
\hline & & $>0.05$ & $<0.05$ & $>0.05$ & $>0.05$ \\
\hline & Restaurant & $\begin{array}{l}1.11(0.82-1.52) \\
>0.05\end{array}$ & $\begin{array}{l}0.88(0.62-1.26) \\
>0.05\end{array}$ & $\begin{array}{l}1.67(1.21-2.29) \\
<0.01\end{array}$ & $\begin{array}{l}1.15(0.76-1.74) \\
>0.05\end{array}$ \\
\hline \multirow{5}{*}{$\begin{array}{l}\text { Place of purchase and } \\
\text { consumption } \\
\text { Canteen/office) }\end{array}$} & \multirow{2}{*}{ Fastfood } & $0.77(0.46-1.28)$ & $0.73(0.42-1.26)$ & $2.36(1.32-4.24)$ & $1.30(0.62-2.70)$ \\
\hline & & $>0.05$ & $>0.05$ & $<0.01$ & $>0.05$ \\
\hline & \multirow{2}{*}{ Street } & $0.92(0.60-1.40)$ & $0.69(0.43-1.09)$ & $0.27(0.17-0.42)$ & $0.43(0.26-0.69)$ \\
\hline & & $>0.05$ & $>0.05$ & $<0.01$ & $<0.01$ \\
\hline & Cafeteria & $\begin{array}{l}0.51(0.29-0.90) \\
<0.05\end{array}$ & $\begin{array}{l}2.06(0.99-4.29) \\
>0.05\end{array}$ & $\begin{array}{l}0.89(0.52-1.52) \\
>0.05\end{array}$ & $\begin{array}{l}1.85(0.78-4.42) \\
>0.05\end{array}$ \\
\hline \multirow{7}{*}{$\begin{array}{l}\text { Fried foods consumption } \\
\text { (Rarely/never) }\end{array}$} & \multirow{5}{*}{ 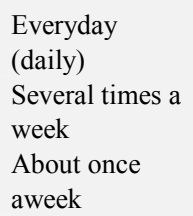 } & $\begin{array}{l}1.25(0.85-1.84) \\
>0.05\end{array}$ & $\begin{array}{l}1.02(0.65-1.58) \\
>0.05\end{array}$ & $\begin{array}{l}2.74(1.76-4.26) \\
<0.01\end{array}$ & $\begin{array}{l}1.80(0.99-3.27) \\
>0.05\end{array}$ \\
\hline & & $0.56(0.37-0.84)$ & $0.53(0.35-0.81)$ & $0.90(0.60-1.34)$ & $0.66(0.40-1.08$ \\
\hline & & $<0.01$ & $<0.01$ & $>0.05$ & $>0.05$ \\
\hline & & $1.23(0.90-1.68)$ & $1.39(0.96-2.00)$ & $0.60(0.43-0.82)$ & $1.10(0.72-1.68)$ \\
\hline & & $>0.05$ & $>0.05$ & $<0.01$ & $>0.05$ \\
\hline & \multirow{2}{*}{ Everymonth } & $0.83(0.41-1.67)$ & $0.80(0.37-1.71)$ & $0.58(0.29-1.16)$ & $0.73(0.31-1.72)$ \\
\hline & & $>0.05$ & $>0.05$ & $>0.05$ & $>0.05$ \\
\hline
\end{tabular}

Dietary habits associated to obesity

The consumption of foods specific to urban environment reveals that consumption of chocolate $[1.83$ (95\% CI: $1.17-$ 2.87)], increased the risk of AO. (Table 6).

Table 6. Binary logistic regression of obesity with the types of food consumed.

\begin{tabular}{|c|c|c|c|c|c|}
\hline \multirow{3}{*}{$\begin{array}{l}\text { Types of food } \\
\text { consumed }\end{array}$} & & Overweight/obesity & GO & AO & $\mathrm{CO}$ \\
\hline & & Odds ratio $(95 \% \mathrm{CI})$ & Odds ratio $(95 \% \mathrm{CI})$ & Odds ratio $(95 \% \mathrm{CI})$ & Odds ratio $(95 \% \mathrm{CI})$ \\
\hline & & P value & P value & P value & $P$ value \\
\hline \multirow{22}{*}{$\begin{array}{l}\text { Foods specific to } \\
\text { urban environment }\end{array}$} & \multirow{2}{*}{ Soft drinks (Yes) } & $0.56(0.38-0.93)$ & $1.17(0.72-1.89)$ & $0.59(0.36-0.95)$ & $0.91(0.51-1.64)$ \\
\hline & & $<0.05$ & $>0.05$ & $<0.05$ & $>0.05$ \\
\hline & \multirow{2}{*}{ Candies (Yes) } & $1.16(0.77-1.74)$ & $1.24(0.80-1.93)$ & $0.67(0.44-1.01)$ & $1.10(0.5-1.84)$ \\
\hline & & $>0.05$ & $>0.05$ & $>0.05$ & $>0.05$ \\
\hline & Chocolate (Yes) & $1.03(0.67-1.58)$ & $0.93(0.59-1.49)$ & $1.83(1.17-2.87)$ & $1.25(0.72-2.18)$ \\
\hline & \multirow{2}{*}{ Chips (Yes) } & $1.10(0.68-1.77)$ & $0.73(0.41-1.28)$ & $0.97(0.57-1.66)$ & $0.63(0.32-1.24)$ \\
\hline & & $>0.05$ & $>0.05$ & $>0.05$ & $>0.05$ \\
\hline & \multirow{2}{*}{$\begin{array}{l}\text { Fried Irish potato } \\
\text { (Yes) }\end{array}$} & $1.09(0.68-1.77)$ & $1.41(0.82-2.41)$ & $1.23(0.75-2.03)$ & $1.78(0.93-3.40)$ \\
\hline & & $>0.05$ & $>0.05$ & $>0.05$ & $>0.05$ \\
\hline & \multirow{2}{*}{ Hamburger (Yes) } & $0.94(0.50-1.78)$ & $0.61(0.30-1.22)$ & $1.11(0.57-2.18)$ & $0.74(0.32-1.72)$ \\
\hline & & $>0.05$ & $>0.05$ & $>0.05$ & $>0.05$ \\
\hline & Hot dog (Yes) & $1.29(0.73-2.28)$ & $1.07(0.57-2.00)$ & $1.25(0.69-2.27)$ & $1.04(0.50-2.17)$ \\
\hline & \multirow{2}{*}{ Pop corn (Yes) } & $1.40(0.91-2.15)$ & $1.58(0.98-2.56)$ & $0.79(0.51-1.23)$ & $1.77(1.00-3.12)$ \\
\hline & & $>0.05$ & $>0.05$ & $>0.05$ & $>0.05$ \\
\hline & \multirow{2}{*}{ Pastries (Yes) } & $0.99(0.64-1.53)$ & $0.63(0.38-1.02)$ & $0.55(0.35-0.86)$ & $0.48(0.27-0.88)$ \\
\hline & & $>0.05$ & $>0.05$ & $<0.01$ & $<0.05$ \\
\hline & \multirow{2}{*}{ Pizza (Yes) } & $0.89(0.44-1.79)$ & $1.011(0.45-2.26)$ & $0.62(0.30-1.31)$ & $0.44(0.18-1.10)$ \\
\hline & & $>0.05$ & $>0.05$ & $>0.05$ & $>0.05$ \\
\hline & \multirow{2}{*}{ Ice cream (Yes) } & $1.47(0.83-2.61)$ & $1.84(0.95-3.56)$ & $1.24(0.67-2.26)$ & $1.83(0.83-4.02)$ \\
\hline & & $>0.05$ & $>0.05$ & $>0.05$ & $>0.05$ \\
\hline & \multirow{2}{*}{$\begin{array}{l}\text { Milk and diary } \\
\text { products (Yes) }\end{array}$} & $1.03(0.70-1.51)$ & $0.67(0.44-1.04)$ & $0.68(0.45-1.02)$ & $0.67(0.40-1.12)$ \\
\hline & & $>0.05$ & $>0.05$ & $>0.05$ & $>0.05$ \\
\hline
\end{tabular}




\begin{tabular}{|c|c|c|c|c|c|}
\hline \multirow{3}{*}{$\begin{array}{l}\text { Types of food } \\
\text { consumed }\end{array}$} & & Overweight/obesity & GO & AO & $\mathrm{CO}$ \\
\hline & & Odds ratio $(95 \% \mathrm{CI})$ & Odds ratio $(95 \% \mathrm{CI})$ & Odds ratio $(95 \% \mathrm{CI})$ & Odds ratio $(95 \% \mathrm{CI})$ \\
\hline & & Pvalue & Pvalue & Pvalue & Pvalue \\
\hline \multirow{16}{*}{ Western foods } & \multirow{2}{*}{ Pastas (Yes) } & $0.96(0.68-1.37)$ & $1.29(0.87-1.92)$ & $1.15(0.79-1.66)$ & $1.17(0.73-1.87)$ \\
\hline & & $>0.05$ & $>0.05$ & $>0.05$ & $>0.05$ \\
\hline & \multirow{2}{*}{ Meat products (Yes) } & $0.77(0.53-1.14)$ & $1.06(0.69-1.62)$ & $0.99(0.67-1.47)$ & $1.04(0.63-1.73)$ \\
\hline & & $>0.05$ & $>0.05$ & $>0.05$ & $>0.05$ \\
\hline & \multirow{2}{*}{ Canned fishes (Yes) } & $0.79(0.56-1.11)$ & $0.87(0.59-1.28)$ & $0.79(0.55-1.11)$ & $0.73(0.46-1.15)$ \\
\hline & & $>0.05$ & $>0.05$ & $>0.05$ & $>0.05$ \\
\hline & \multirow{2}{*}{$\begin{array}{l}\text { Canned vegetables } \\
\text { (Yes) }\end{array}$} & $0.97(0.63-1.50)$ & $0.95(0.58-1.55)$ & $1.06(0.68-1.66)$ & $1.15(0.64-2.07)$ \\
\hline & & $>0.05$ & $>0.05$ & $>0.05$ & $>0.05$ \\
\hline & \multirow{2}{*}{ Cheese (Yes) } & $1.19(0.79-1.81)$ & $1.47(0.92-2.36)$ & $0.899(0.59-1.38)$ & $1.22(0.71-2.12)$ \\
\hline & & $>0.05$ & $>0.05$ & $>0.05$ & $>0.05$ \\
\hline & \multirow{2}{*}{ Salad (Yes) } & $0.63(0.44-0.89)$ & $0.98(0.66-1.45)$ & $0.80(0.56-1.14)$ & $0.91(0.57-1.45)$ \\
\hline & & $<0.05$ & $>0.05$ & $>0.05$ & $>0.05$ \\
\hline & \multirow{2}{*}{ Shawarma (Yes) } & $1.38(0.87-2.18)$ & $0.95(0.56-1.62)$ & $1.86(1.14-3.03)$ & $1.49(0.76-2.91)$ \\
\hline & & $>0.05$ & $>0.05$ & $>0.05$ & $>0.05$ \\
\hline & \multirow{2}{*}{$\begin{array}{l}\text { Rice with soup } \\
\text { (Yes) }\end{array}$} & $0.65(0.43-0.96)$ & $0.55(0.34-0.88)$ & $0.41(0.26-0.63)$ & $0.34(0.18-0.63)$ \\
\hline & & $<0.05$ & $<0.05$ & $<0.01$ & $>0.05$ \\
\hline \multirow{4}{*}{$\begin{array}{l}\text { Local dishes and } \\
\text { fruits }\end{array}$} & \multirow{2}{*}{ Local dishes (No) } & $0.57(0.41-0.79)$ & $0.82(0.57-1.18)$ & $1.36(0.98-1.89)$ & $0.85(0.55-1.30)$ \\
\hline & & $<0.01$ & $\mathrm{P}>0.05$ & $\mathrm{P}>0.05$ & $\mathrm{P}>0.05$ \\
\hline & \multirow{2}{*}{ Fruits (No) } & $1.04(0.73-1.47)$ & $1.12(0.75-1.68)$ & $1.28(0.89-1.84)$ & $1.00(0.62-1.60)$ \\
\hline & & $\mathrm{P}>0.05$ & $\mathrm{P}>0.05$ & $\mathrm{P}>0.05$ & $\mathrm{P}>0.05$ \\
\hline
\end{tabular}

Table 7. Binary logistic regression of obesity with tobacco and alcohol consumption.

\begin{tabular}{llllll}
\hline \multirow{5}{*}{} & & Overweight/obesity & GO & AO & CO \\
\cline { 3 - 6 } & & Odds ratio (95\%CI) & Odds ratio (95\%CI) & Odds ratio (95\%CI) & Odds ratio (95\%CI) \\
\cline { 3 - 6 } & P value & P value & P value & P value \\
\hline \multirow{6}{*}{ Alcohol } & Ordinary or Imported & $0.71(0.49-1.02)$ & $1.226(0.81-1.86)$ & $1.10(0.75-1.61)$ & $1.59(0.98-2.60)$ \\
& beers (Yes) & $>0.05$ & $>0.05$ & $>0.05$ & $>0.05$ \\
& Imported Wines and & $0.80(0.54-1.20)$ & $0.69(0.44-1.082)$ & $0.76(0.50-1.16)$ & $0.79(0.46-1.34)$ \\
& apperitifs (Yes) & $>0.05$ & $>0.05$ & $>0.05$ & $>0.05$ \\
& Imported distilled & $1.07(0.72-1.61)$ & $1.23(0.79-1.91)$ & $1.67(1.10-2.54)$ & $1.23(0.72-2.10)$ \\
& beverages (Yes) & $>0.05$ & $>0.05$ & $<0.05$ & $>0.05$ \\
\multirow{2}{*}{ Tobacco } & Cigarette smoking & $0.98(0.59-1.63)$ & $1.59(0.86-2.93)$ & $3.25(1.83-5.78)$ & $2.59(1.15-5.79)$ \\
& (Yes) & $>0.05$ & $>0.05$ & $<0.01$ & $<0.05$ \\
\hline
\end{tabular}

For western foods, traditional foods and fruits, it appears that consumption of salad $(\mathrm{OR}=0.63 ; 95 \% \mathrm{CI}$ : $0.44-0.89)$; rice and soup $(\mathrm{OR}=0.65 ; 95 \% \mathrm{CI}$ : $0.43-0.96)$ and non consumption of traditional foods $(\mathrm{OR}=0.57$; $95 \% \mathrm{CI}$ : $0.41-$ $0.79)$ protect against overweight/obesity. The consumption of rice and soup was also protective against GO $[0.55$ (95\% CI: 0.34-0.88)] and AO [0.41 (95\% CI: 0.26-0.63)] (Table 6).

Concerning alcohol and tobacco consumption, that the consumption of imported distilled beverages increased by 1.67 (95\% CI: 1.10-2.54) times the risk of AO smoking tobacco increased by 3.25 (95\% CI: $1.83-5.78)$ the risk of developing $\mathrm{AO}$ and by 2.59 those of having $\mathrm{CO}$ (95\% CI: 1.15-5.79) as show in Table 7.

\section{Discussion}

\subsection{Sociodemographic Factors}

The etiology of obesity is multifactorial, involving complex interactions among the genetic background, hormones and different social and environmental factors, such as sedentary lifestyle and unhealthy dietary habits [22].

In Africa, the high prevalence of obesity has been attributed to demographic and nutritional transition that have resulted from urbanization and the westernization of lifestyle behaviours [23], which include lack of physical activities and changes in food habits [24]. The direct consequences are CVD, diabetes mellitus, osteoarthritis, dyslipidemia, and cancer [25]. Our study recorded high prevalence of all types of obesity (overweight/obesity, GO, $\mathrm{AO}$ and $\mathrm{CO}$ ) among Douala dwellers.

The study suggested that the prevalence of all types of obesity increases with age but age category 38-47 was associated to the occurrence of all types of obesity. Many studies conducted in developed and developing countries indicated that mean body weight and BMI gradually increase during late adulthood life [26]. This is likely due to natural changes in body composition and decreasing rate of metabolism associated with aging [27].

Concerning gender, the study found high prevalence of all type of obesity among women compared to men and the risk of being obese was greater among women than men varies from 1.45 (overweight/ obesity) to 14.48 (AO). This is in accordance with results found in many developing countries [28]. This difference in the prevalence of obesity between women and men will have biological basis. Women, in general, have a higher percentage of body fat than men and their resting fat metabolism is lower than in men [29]. Another possible explanation is the increased adoption of sedentary lifestyles and reduction of physical exercises [30] 
in women compared to men. In developing countries males are more frequently engage in physically demanding activities than women; hence they may have reduced risk of obesity.

Concerning marital status, marriage (or in couple) was associated with all types of obesity, this is in accordance with Sobal et al., [31] who noted that married people are more often overweight and obese than those living alone. There are three major perspectives linking body weight to marital status. In fact, married individuals are more likely to have a confidant with whom to eat and may therefore eat more regularly, leading to weight gain [32]. Also, Married men and women are less likely to be conscious of or concerned about their body weight because they are not actively seeking a mate [33]. As a consequence, married individuals are more likely to experience greater increase in body weight than comparable non-married individuals who are attempting to minimize weight gain in order to attract a partner. In addition, other research showing that women are more likely to take care of men rather than the reverse suggests that the marriage market effect is more important for women [34].

Educational level is used in many epidemiological studies as a valuable indicator of socioeconomic position. The accumulated evidence suggests an inverse association between education level and obesity mostly among women in developed societies while in men this association is less consistent [35]. In terms of education level, our results indicate that secondary level was strongly associated to all types of obesity, primary level was strongly associated to GO and $\mathrm{CO}$ and university level was associated to overweight/obesity, GO and CO. Higher educational status may result in changes in lifestyle with adoption of sedentary lifestyle practices (motorized transportation, sedentary employment...) and ability to eat in restaurants in general. However, inverse associations between education and obesity have been found in other studies [36]. Persons with higher educational status may be well informed to change their lifestyles and to adopt healthy lives.

In terms of source of incomes, our results indicate that trade activity increased 1.43 times overweight/obesity, 1.74 times $\mathrm{AO}$ and 1.73 times $\mathrm{CO}$. The causes of obesity among traders are not farfetched as these groups of people are mostly engaged in sedentary activities, coupled with long hours of physical inactivity and high energy intake [37].

\subsection{Practical Lifestyle Habits}

Results from our study support the hypothesis that eating patterns are associated with obesity. Studies have suggested that several characteristics of dietary behaviours such as eating frequency, the distribution of food consumption across the day, breakfast skipping, and the frequency of meals eaten away from home, together referred to as "eating patterns," may influence body weight [38].

In our study, eating two meals per day increased 1.51 times (95\% CI: 1.06-2.15) AO and eating meals morning and evening increased 3.12 times (95\% CI: 1.99-4.89) AO.

It has demonstrated that low meal frequency is associated with higher 24-hour insulin concentrations. On the other hand, eating multiple, small meals may suppress hunger and overall serum insulin concentrations. Insulin inhibits lipase enzyme activity and increases fat deposition. Since insulin is related to fatty acid storage, meal frequency maybe one of the factors affecting body weight [39].

One could suppose that individuals who do not eat early in the day may tend to be hungry later and then may consume a greater number of calories in the evening than individuals who eat consistently throughout the day. Greater energy intake may result in greater fat storage and, thus, may be one of the factors leading to obesity [39].

Eating late in the evening may increase the amount of muscle glycogen. In humans, muscle glycogen fluctuates with muscle activity and subsequent carbohydrate consumption. Data suggest that the consumption of carbohydrate-rich foods in the late evening leads to increased glycogen levels in the muscles. Unless this stored glycogen is burned as fuel, excess will ultimately be stored as fat. Therefore, consumption of late-evening meals with carbohydrate-rich foods may also be related to obesity through its effect on hormonal regulation of energy and lipid metabolism [38].

Away-from-home foods consumption is an important determinant of dietary intake and risk for obesity. Our study revealed that away-from-home foods consumption increased 1.69 times (95\% CI: 1.07-2.68) GO. In addition, consumption of foods in restaurants and fast foods was strongly associated to AO in our study.

Current research reveals that several environmental factors could be fuelling the obesity epidemic; diet quality is coming to the fore as a major modifiable factor. In our modern fastpaced lives, less time is dedicated to the preparation of meals [40]. The same phenomenon is observed in the big Cameroonian cities like Douala where eating in restaurants and fast foods would become normal. The stated reasons would be for example the European model appreciated by the population, the busy schedules for some and the cooking facilities for others, the affordable prices for people of low economic level. With the advent of large European supermarkets, fast food restaurants and cheap restaurants abound. The dishes proposed are cooked and embrace the practices and culinary presentations of the different regions of Cameroon.

The consumption of fast foods or meals outside the home has consistently been linked to the consumption of more energy, saturated fat and sodium and fewer fruits and vegetables [41], fiber, calcium, and iron than foods prepared at home [42]. A positive association between eating out of home and weight gain has been confirmed by two systematic reviews [43].

In our study, the results of fried foods consumption have revealed a high prevalence of obesity. Also, daily consumption increased by 2.74 times (95\% CI: $1.76-4.26)$ the risk of $\mathrm{AO}$, several times a week consumption was associated to overweight/obesity and GO and about once a week consumption was associated to $\mathrm{AO}$. In fact, frying 
process food loses water and absorbs oil up to $20 \%$ of its weight and become more energetic, hence [44]. Several mechanisms may explain a positive association between fried food intake and obesity. Fried foods are crunchy, aromatic, highly palatable, and rich in fats. As a consequence, eating fried food in ad libitum conditions may result in higher absolute intake of foods with high energy density and low satiety index. The relatively low satiety index of fats may be related to their low ability to stimulate insulin and leptin production [44]. Fried food may also induce obesity by increasing food energy density. Fats are absorbed and metabolized more efficiently than are other macronutrients [45].

\subsection{Dietary Behaviours}

In our study, chocolate consumption increased 1.86 times the risk of (95\% CI: 1.17-2.87) AO.

Carbohydrate foods low in fiber like chocolate but high in free sugars and / or sugar added, in addition to their contribution to energy density and possible conversion lipids (glycogen to glucose and then to free fatty acids and triglycerides) would be also involved in various metabolic disorders because of their effect hyperglycemia resulting from their high glycemic index [46]. The excessive consumption of foods with a high glycemic index and glycemic load (potatoes, refined cereals, sugary soft drinks, etc.) is indeed associated with the metabolic disorders responsible for these chronic diseases [47].

\subsection{Alcohol and Cigarette Smoking}

Alcohol consumption is relatively high in Cameroon [48]. The results of this study show that imported distilled beverages consumers has 1.67 times (95\% CI: $1.10-2.54)$ increase odds of AO. Research suggests that alcohol consumption may represent a sizable risk factor for weight gain [49]. The association, posited to be a product of differential drink type preference, is typically strongest among men [50] who are more likely to favor beer, which is carbohydrate rich [51]. Alcohol, which is the second most energy dense macronutrient consumed, is known to reduce oxidation of fat and favors fat storage which may result in weight gain. However, previous studies have shown that despite the added calories, alcohol drinkers do not gain extra weight as compared to non-drinkers [52].

Cigarette smoking is one of the leading causes of preventable morbidity and mortality [53]. Data on the prevalence of smoking in Cameroon are scarces. Unpublished data from a national survey indicate that the overall prevalence of smoking is approximately $6.4 \%$ in Cameroon [48].

Our study revealed that, cigarette smoking significantly increased odds of AO [3.25 (95\% CI: 1.83-5.78)] and CO [2.59 (1.15-5.79)]. Several studies have indicated that smoking behaviour is closely related to body weight and obesity [54], and shown that body weight of past smokers was heavier than that of never smokers [55]. The mechanisms underlying the impact of smoking on weight include varying energy intake, physical activity, metabolic rate, and inflammatory status linked to smoking status [54]. Also, it has been reported that smoking affects fat distribution in the abdominal area by various biological mechanisms such as the dysregulation of the hypothalamic-pituitary-adrenal axis [56]. Another biological mechanism between smoking and increased abdominal obesity is that smoking affects the regulation of the sex hormones [57]. Moreover, smoking may increase insulin resistance [58].

\section{Strengths and Limitations}

The results of our study are difficult to generalize because the study was only carried out on a sample of the inhabitants of the city of Douala. Cameroon has ten regions and several villages with totally different environmental and cultural specificities.

Moreover, this work makes it possible to challenge all the stakeholders involved in the health field that obesity must not only be considered as a risk factor for CVD but also as an emerging disease influenced by several factors including unhealthy diet. This work also makes it possible to complete the policies for the control and prevention of noncommunicable diseases in Cameroon.

Conclusion: The prevalence of obesity is high in Douala. Several profiles of obesity have been identified and the prevalence of each type of obesity is high. Several factors are associated and tend to explain this prevalence. a sustainable approach towards effective prevention is a more affordable strategy by adopt combined lifestyle interventions targeting physical activity as well as diet and environmental factors (economic, sociocultural and physical environments). More research should be carry out to generate datas on obesity differences among urban and rural areas.

\section{Availability of Data and Materials}

The primary data and materials of this study are available from the corresponding author. Official registration is required to access the database. The data sets analyzed during the study are available from the corresponding author.

\section{Authors' Contributions}

CFNB and designed the study protocol and wrote the first manuscript draft. NBCF and FRN led the statistical analyses and contributed to the manuscript drafting. NM, JN and MNM contributed to data collection. NBCF, WD and FRN critically contributed to the analysis, discussion and interpretation of the data and contributed to data interpretation and the writing of the manuscript. All authors reviewed and approved the final manuscript draft. 


\section{Ethics Approval and Consent to Participate}

The study protocol was approved by the National Ethics Committee $\mathrm{N}^{\circ} 2014 / 08 / 488 / \mathrm{EC} / \mathrm{CNERSH}$ and was conducted in strict compliance with the physical, moral and psychological integrity of all participants; following the principles outlined in the Helsinki Declaration. Prior to participant enrolment, they were informed about the purpose of the study and data collection procedures and all volunteer participants signed an informed consent form.

\section{Consent for Publication}

Not applicable.

\section{Conflict of Interest Statement}

The authors declare that they have no competing interests.

\section{Acknowledgements}

The authors express their grateful thanks to the Cameroon Nutritional Science Society and to all the participants to the survey.

\section{References}

[1] Rajkamal R, Singh Z, Stalin P, Muthurajesh E (2015). Prevalence and determinants of overweight and obesity among elderly population in an urban area of Puducherry. Intern. $J$. Med. Sci. and Public Health. 4: 3.

[2] Zou Y, Zhang R, Zhou B, Huang L, Chen J, Gu F, Zhang H, Fang Y, Ding G (2015). A comparison study on the prevalence of obesity and its associated factors among city, township and rural area adults in China. BMJ Open. 5: e008417. doi: 10.1136/bmjopen-2015-008417.

[3] Guh DP, Zhang W, Bansback N, et al (2009). The incidence of co-morbidities related to obesity and overweight: a systematic reviewand meta-analysis. BMC Public Health; 9: 88.

[4] Flegal KM, Kit BK, Orpana H, Graubard BI (2013). Association of all-cause mortality with overweight and obesity using standard body mass index categories: a systematic review and meta-analysis. JAMA 309: 71-82.

[5] World Health Organization (WHO) (2009). Global health risks mortality and burden of disease attributable to selected major risks. Geneva: Switzerland.

[6] Ge W. et al. (2014). Association between anthropometric measures of obesity and subclinical atherosclerosis in Bangladesh. Atherosclerosis 232, 234-241.

[7] Cai L., Liu A., Zhang Y. \& Wang P. (2013). Waist-to-height ratio and cardiovascular risk factors among Chinese adults in Beijing. PLoS One 8, e69298.

[8] Guasch-Ferre M. et al. (2012). Waist-to-height ratio and cardiovascular risk factors in elderly individuals at high cardiovascular risk. PLoS One 7, e43275.
[9] Wannamethee SG, Shaper AG, Walker M (2005). Overweight and obesity and weight change in middle aged men: impact on cardiovascular disease and diabetes. J Epidemiol Community Health; 59: 134-139.

[10] Fezeu L, Balkau B, Sobngwi E, Kengne AP, Vol S, Ducimetiere P and Mbanya JC (2010). Waist circumference and obesity-related abnormalities in French and Cameroonian adults: the role of urbanization and ethnicity. Int J Obes 34, 446-453.

[11] Stone RE, Nankap M, Ndjebayi AO, Friedman A, Tarini A, Brown KH, Kaiser L (2018). Prevalence and predictors of overweight and obesity among Cameroonian women in a national survey and relationships with waist circumference and inflammation in Yaoundé and Douala. Matern Child Nutr. 14: e12648. https://doi.org/10.1111/mcn.12648.

[12] Correia J, Pataky Z, Golay A (2014). Comprendre l'obésité en Afrique: poids du développement et des représentations. Rev Med Suisse 10: 712-6.

[13] Downey, G (2016). Being human in cities: Phenotypic bias from urban niche construction. Curr. Anthropol. 57, 52-64.

[14] Ebrahim, S.; Pearce, N.; Smeeth, L.; Casas, J. P.; Jaffar, S.; Piot, P (2013). Tackling non-communicable diseases in lowand middle-income countries: Is the evidence from highincome countries all we need? PLoS Med. 10, e1001377.

[15] Mapa-Tassou C., Bonono C. R., Assah F., Ongolo-Zogo P., Sobngwi E., Mbanya J. C. (2017): Analysis of noncommunicable diseases prevention policies in Africa (ANPPA): Cameroon Case Study. A Technical Research Report developed for the African Population \& Health Research Centre (APHRC).

[16] Swinburn BA, Sacks G, Hall KD, McPherson K, Finegood DT, Moodie ML, et al (2011): The global obesity pandemic: shaped by global drivers and local environments. Lancet; 378: 804-814.

[17] Glass TA, McAtee MJ (2006): Behavioral science at the crossroads in public health: extending horizons, envisioning the future. Soc Sci Med; 62: 1650-1671.

[18] Van Dis I, Kromhout D, Geleijnse JM, Boer JM, Verschuren WM (2009). Body mass index and waist circumference predict both 10-year nonfatal and fatal cardiovascular disease risk: study conducted in 20,000 Dutch men and women aged 20-65 years. Eur J Cardiovasc Prev Rehabil. 16 (6): 729-34.

[19] Ahmed SH, Meyer HE, Kjøllesdal MK, Marjerrison N, Mdala I, Htet AS, Bjertness E and Madar AA (2019): The prevalence of selected risk factors for non-communicable diseases in Hargeisa, Somaliland: a cross-sectional study. BMC Public Health 19: 878 .

[20] NCEP (2001). Expert panel on detection, evaluation and treatment of high blood cholesterol in adults (Adult treatment panel III). Executive summary of the third report on the National cholesterol education program (NCEP). JAMA. 285 (19): 2486-2497.

[21] Pradeepa R, Anjana RM, Joshi SR, Bhansali A, Deepa M, Joshi PP, Dhandania VK, Madhu SV, Rao PV, Geetha L, Subashini R, Unnikrishnan R, Shukla DK, Kaur T, Mohan V andDas $\mathrm{AK}$, for the ICMR-INDIAB Collaborative Study Group (2015). Prevalence of generalized \& abdominal obesity in urban \& rural India- the ICMR-INDIAB Study (Phase-I) [ICMR-INDIAB-3]. Indian $J$ Med Res 142, 139-150 DOI: 10.4103/0971-5916.164234. 
[22] Chan RSM and Woo J (2010). Prevention of overweight and obesity: how effective is the current public health Approach. Int J Environ Res Public Health 7 (3): 765-783.

[23] Steyn, NP., Nel, JH., Parker, WA., Ayah, R., Mbithe, D (2011). Dietary, social, and environmental determinants of obesity in Kenyan women. Scand J Public Health 39: 88-97.

[24] Baalwa, J., Byarugaba, BB., Kabagambe, EK., Otim, AM (2010). Prevalence of overweight and obesity in young adults in Uganda. Afr Health Sci. 10: 367-73.

[25] Cappuccio, FP., Kerry, SM., Adeyemo, A., Luke, A., Amoah, AG., Bovet, P. et al (2008). Body size and blood pressure: An analysis of Africans and the African diaspora. Epidemiology19: 38-46.

[26] Jafar TZ, Chaturvedi N, Pappas G (2006). Prevalence of overweight and obesity and their association with hypertension and diabetes mellitus in an indo-Asian population. CMAJ. 175 (9): 1071-7.

[27] Villareal DT, Apovian CM, Kushner RF, Klein S (2005). Obesity in older adults: technical review and position statement of the American Society for Nutrition and NAASO, the Obesity Society. Am J ClinNutr. 82 (5): 923-34.

[28] Rengma MS, Sen J, Mondal N (2015). Socio-economic, demographic and lifestyle determinants of overweight and obesity among adults of Northeast India. Ethiop J Health Sci. 25 (3): 199-208.

[29] Toth, MJ., Gardner, AW., Arciero, PJ., Calles-Escandon, J., Poehlman, ET (1998). Gender differences in fat oxidation and sympathetic nervous system activity at rest and during submaximal exercise in older individuals. Clin Sci. 95: 59-66.

[30] Hu, FB (2003). Sedentary lifestyle and risk of obesity and type 2 diabetes. Lipids 38: 103-8.

[31] Sobal J, Hanson KL, Frongillo EA (2009): Gender, Ethnicity, Marital Status, and Body Weight in the United States. Obesity (Silver Spring), 17 (12): 2223-2231.

[32] Averett S, Argys L, Sorkin J (2012). In sickness and in health: An examination of relationship status and health using data from the Canadian national public health survey. Review of Economics of the Household. Advance online publication. 10.1007/s11150-012-9143-Z

[33] Wilson S (2012). Marriage, gender, and obesity in later life. Economics \& Human Biology. 10: 431-453. [PubMed: 22795874]

[34] Umberson D, Liu H, Powers D (2009). Marital status, marital transitions, and body weight. Journal of Health and Social Behavior. 50: 327-343. [PubMed: 19711809].

[35] Vernay M, Malon A, Oleko A, Salanave B, Roudier C, Szego E, Deschamps V, Hercberg S, Castetbon K (2009): Association of socioeconomic status with overall overweight and central obesity in men and women: the French Nutrition and Health Survey 2006. BMC Public Health, 9: 215.

[36] Hajian-Tilaki, KO., Heidari, B (2010). Association of educational level with risk of obesity and abdominal obesity in Iranian adults. $J$ Public Health (Oxf); 32: 202-9.

[37] Afolabi WA, Addo AA, Sonibare MA (2004). Activity pattern, energy intake and obesity among Nigerian urban market women. Int J Food SciNutr. 55 (2): 85-90.
[38] Wyatt HR, Grunwald GK, Mosca CL, et al. (2002). Long-term weight loss and breakfast in subjects in the National Weight Control Registry. Obes Res 10: 78-82.

[39] Ma Y, Bertone ER, Stanek III EJ, Reed GW, Hebert JR, Cohen NL, Merriam PA, and Ockene IS (2003). Association between Eating Patterns and Obesity in a Free-living US Adult Population. Am J Epidemiol. 158: 85-92.

[40] Monteiro CA, Levy RB, Claro RM, et al (2010). A new classification of foods based on the extent and purpose of theirprocessing. CadSaude Publica 26, 2039-2049.

[41] O'Donnell SI, Hoerr SL, Mendoza JA, et al.(2008). Nutrient quality of fast food kids meals. Am J ClinNutr 88, 1388-1395.

[42] Guthrie JF, Lin BH, et al (2002). Role of food prepared away from home in the American diet, 1977-78 versus 1994-96: changes and consequences. Journal of Nutrition Education and Behavior. 34 (3): 140-150. [PubMed: 12047838].

[43] Rosenheck R (2008). Fast food consumption and increasedcaloric intake: a systematic review of a trajectory towards weight gain and obesity risk. Obes Rev9, 535-547.

[44] Guallar-Castillon P, Rodríguez-Artalejo F, Fornés NS, Banegas JR, Etxezarreta PA, Ardanaz E, Barricarte A, Chirlaque MD, Iraeta MD, Larranaga NL, Losada A, Mendez M, Martínez C, Quiros JR, Navarro C, Jakszyn P, Sanchez MJ, Tormo MT, and Gonzalez CA (2007). Intake of fried foods is associated with obesity in the cohort of Spanish adults from the European Prospective Investigation into Cancer and Nutrition. Am J Clin Nutr; 86: 198-205

[45] Dourmashkin JT, Chang GQ, Gayles EC, et al (2005). Different forms of obesity as a function of diet composition. Int J Obes (Lond); 29: 1368-78.

[46] Ludwig DS (2002). The Glycemic Index: Physiological Mechanisms Relating to Obesity, Diabetes, and Cardiovascular Disease. JAMA. 287 (18): 2414-23.

[47] Hodge AM, English DR, O'Dea K, Giles GG (2004). Glycemic Index and Dietary Fiber and the Risk of Type 2 Diabetes. Diabetes Care 27 (11): 2701-6.

[48] Echouffo Tcheugui JB and Kengne AP (2011). Chronic noncommunicable diseases in Cameroon - burden, determinants and current policies. Globalization and Health 7: 44.

[49] Tolstrup JS, Heitmann BL, Tjønneland AM, et al (2005). The relation between drinking pattern and body mass index and waist and hip circumference. Int J Obes (Lond). 29 (5): 490497.

[50] French MT, Norton EC, Fang H, Maclean JC (2010). Alcohol consumption and body weight. Health Econ. 19 (7): 814-832.

[51] Shelton N, Savell E (2011). The geography of binge drinking: the role of alcohol-related knowledge, behaviours and attitudes. Results from the Health Survey for England 2007. Health Place. 17 (3): 784---792.

[52] Arif AA and Rohrer JE (2005). Patterns of alcohol drinking and its association with obesity: data from the third national health and nutrition examination survey, 1988-1994. BMC Public Health. 5: 126. 
[53] Centers for Disease Control and Prevention (US), National Center for Chronic Disease Prevention and Health Promotion (US), Office on Smoking and Health (US) (2010). How Tobacco Smoke Causes Disease: The biology and behavioral basis for smoking-attributable disease: A Report of the Surgeon General. Atlanta (GA): Centers for Disease Control and Prevention (US).

[54] Filozof C, Fernandez Pinilla MC, Fernandez-Cruz A (2004). Smoking cessation and weight gain. Obes Rev 5 (2): 95-103. PMID: 15086863.

[55] Mackay DF, Gray L, Pell JP (2013). Impact of smoking and smoking cessation on overweight and obesity: Scotland-wide, cross-sectional study on 40,036 participants. BMC Public Health 15 (13): 348-356.

[56] Audrain-Mcgovern, J., Benowitz, N. L. (2011). Cigarette smoking, nicotine, and body weight. Clin. Pharmacol. Ther. 90 (1), 164-168.http://dx.doi.org/10.1038/clpt.2011.105.

[57] Chiolero, A., Faeh, D., Paccaud, F., Cornuz, J. (2008). Consequences of smoking for body weight, body fat distribution, and insulin resistance. Am. J. Clin. Nutr. 87 (4), 801-809 (pmid: 18400700).

[58] Cena, H., Fonte, M. L., Turconi, G. (2011). Relationship between smoking and metabolic syndrome. Nutr. Rev. 69 (12), 745-753. http://dx.doi.org/10.1111/j.1753-4887.2011.00446.x. 